\title{
RADIATION FROM ACCELERATED PARTICLES IN RELATIVISTIC JETS WITH SHOCKS, SHEAR-FLOW, AND RECONNECTION
}

\author{
K.-I. Nishikawa ${ }^{1}$, B. Zhang ${ }^{2}$, I. Dutan ${ }^{3}$, M. Medvedev ${ }^{4}$, P. Hardee ${ }^{5}$, \\ E.J. Choi ${ }^{6}$, K.W. Min ${ }^{6}$, J. Niemiec ${ }^{7}$, Y. Mizuno ${ }^{8}$, A. Nordlund ${ }^{9}$, \\ J.T. Frederiksen ${ }^{9}$, H. Sol ${ }^{10}$, M. Pohl ${ }^{11}$ and D.H. Hartmann ${ }^{12}$
}

\begin{abstract}
We investigated particle acceleration and shock structure associated with an unmagnetized relativistic jet propagating into an unmagnetized plasma. Strong magnetic fields generated in the trailing shock contribute to the electrons transverse deflection and acceleration. We have calculated, self-consistently, the radiation from electrons accelerated in these turbulent magnetic fields. We found that the synthetic spectra depend on the bulk Lorentz factor of the jet, its temperature and strength of the generated magnetic fields. We have also investigated accelerated electrons in strong magnetic fields generated by kinetic shear (Kelvin-Helmholtz) instabilities. The calculated properties of the emerging radiation will guide our understanding of the complex time evolution and/or spectral structure in gamma-ray bursts, relativistic jets in general, and supernova remnants.
\end{abstract}

${ }^{1}$ Center for Space Plasma and Aeronomic Research, University of Alabama in Huntsville, 320 Sparkman Drive, ZP12, Huntsville, AL 35805, USA

2 Department of Physics, University of Nevada, Las Vegas, NV 89154, USA

${ }^{3}$ Institute of Space Science, Atomistilor 409, Bucharest-Magurele 077125, Romania

${ }^{4}$ Department of Physics and Astronomy, University of Kansas, KS 66045, USA

${ }^{5}$ Department of Physics and Astronomy, The University of Alabama, Tuscaloosa, AL 35487, USA

${ }^{6}$ Korea Advanced Institute of Science and Technology, Daejeon 305-701, South Korea

7 Institute of Nuclear Physics PAN, ul. Radzikowskiego 152, 31-342 Kraków, Poland

8 Institute of Astronomy National Tsing-Hua University, Hsinchu, Taiwan 30013, R.O.C

${ }^{9}$ Niels Bohr Institute, University of Copenhagen, Juliane Maries Vej 30, 2100 Copenhagen, Denmark

${ }^{10}$ LUTH, Observatoire de Paris-Meudon, 5 place Jules Jansen, 92195 Meudon Cedex, France

${ }^{11}$ Institue of Physics and Astronomy, University of Potsdam, Karl-Liebknecht-Strasse 24/25, 14476 Potsdam-Golm, Germany

12 Department of Physics and Astronomy, Clemson University, Clemson, SC 29634, USA 


\section{Simulation of kinetic Kelvin-Helmholz instability}

Recent kinetic simulations have focused on magnetic field generation via electromagnetic plasma instabilities in unmagnetized flows without velocity shears. Three-dimensional (3D) particle-in-cell (PIC) simulations of Weibel turbulence (Nishikawa et al. 2009) have demonstrated subequipartition magnetic field generation. We have calculated, self-consistently, the radiation from electrons accelerated in the turbulent magnetic fields. We found that the synthetic spectra depend on the Lorentz factor of the jet, its thermal temperature and strength of the generated magnetic fields (Nishikawa et al. 2011, 2012). These works have neglected the role of velocity shear in the flow, which are an alternative mechanism to generate subequipartition magnetic fields in relativistic outflows (Alves et al. 2012). Furthermore, a shear flow upstream of a shock can lead to density inhomogeneities via the Kelvin-Helmholtz instability (KHI) which may constitute important scattering sites for particle acceleration.

We have performed simulations with a relativistic core jet surrounded by a sheared velocity layer with the stationary sheath plasmas as simulated in our RMHD simulations (Mizuno et al. 2007). In our initial simulations the initial conditions for the shear flow will have a core jet with $v_{\text {core }}=0.9978 c(\gamma=15)$ pointing in the positive $x$ direction in the middle of the simulation box as in Alves et al. (2012); the upper and lower quarter of the simulation box will contain a sheath also moving in the positive $x$ direction with $v_{\text {sheath }}=0$ (for this simulation). Overall, this structure is similar in spirit, although not in scale, to that proposed for active galactic nuclei (AGN) relativistic jet cores surrounded by a slower moving sheath, and is also relevant to gamma-ray burst (GRB) jets. In particular, we note that this structure is also relevant to the "jet-in-a-jet" or "needles" in a jet scenarios (Giannios et al. 2009, papers therein) which have been invoked to provide smaller scale high speed structures within a much larger more slowly moving AGN jet. Similar smaller scale structures within GRB jets are also conceivable.

This more realistic setup is different from the initial conditions used by the previous simulations with counter-steaming flow of Alves et al. (2012), and hence allows us to compute synthetic spectra in the observer frame far along the $x$-direction. As discussed by Alves et al. (2012), in our setup the growing kinetic KHI will propagate with the flow. For GRB jets, the relativistic jet core will have much higher density relative to the external medium. On the other hand, for an AGN the relativistic core is less dense than the surrounding sheath.

We have performed simulations using a system with $\left(L_{\mathrm{x}}, L_{\mathrm{y}}, L_{\mathrm{z}}\right)=(1005 \Delta$, $205 \Delta, 205 \Delta)$ with the mass ratio of ion and electron, $m_{\mathrm{i}} / m_{\mathrm{e}}=20$. Figure 1 shows the magnetic field structures generated by shearing relativistic electronion flows with $\gamma=15$ with stationary sheath plasmas taken at time $t=70 \omega_{\mathrm{pe}}^{-1}$. Figure 1a shows the magnetic field intensity of $B_{\mathrm{y}}$ plotted in the $y-z$ plane at the center of the box $x=500 \Delta$ (jet out of the plane) with the magnetic finds $B_{\mathrm{y}}$ (red), $B_{\mathrm{x}}$ (black), and $B_{\mathrm{z}}$ (blue) at $x=500 \Delta$ and $y=100 \Delta$. Figure $1 \mathrm{c}$ shows the $x$ component of current. The relativistic jet is directed out of the plane and the positive current is generated at the jet side, whereas the negative current is 

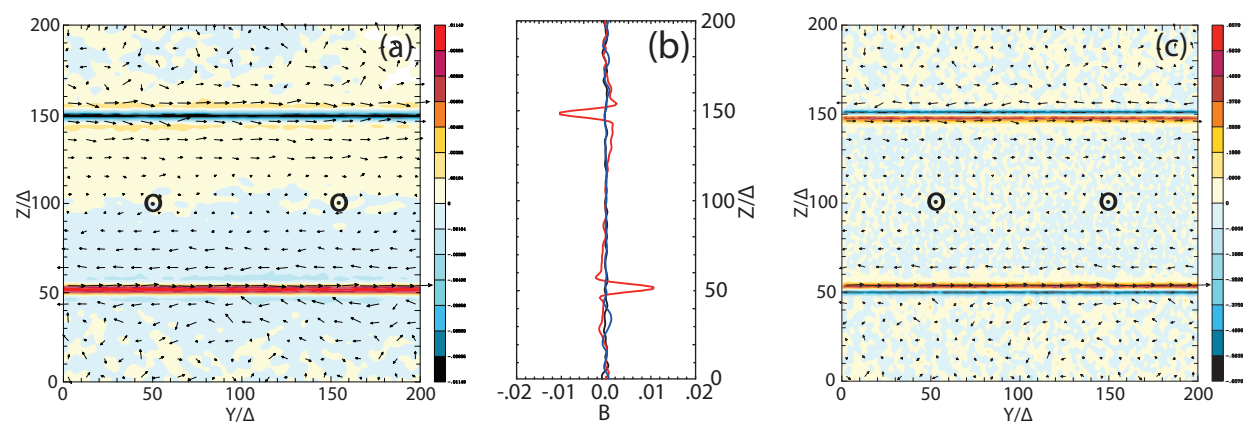

Fig. 1. Magnetic field structures generated by shearing relativistic electron-ion flows with $\gamma=15$ with stationary sheath plasmas taken at time $t=70 \omega_{\mathrm{pe}}^{-1}$. Here $\omega_{\mathrm{pe}}$ is the electron plasma frequency. The magnetic field intensity of $B_{\mathrm{y}}$ is plotted in the $y-z$ plane at the center of the box $x=500 \Delta$ (a) (jet out of the plane). Figure 1b shows the magnetic fields $B_{\mathrm{y}}$ (red), $B_{\mathrm{x}}$ (black), and $B_{\mathrm{z}}$ (blue) at $x=500 \Delta$ and $y=100 \Delta$. Figure $1 \mathrm{c}$ shows the $x$ component of the current density. The relativistic jet is directed out of the plane and the positive current is generated at the core jet side and the negative current is generated in the sheath side. The positive currents are stronger than the negative ones, therefore the $B_{\mathrm{y}}$ components are generated as shown in Figure $1 \mathrm{~b}$.

generated in the sheath side. The positive currents are stronger than the negative currents, therefore the $B_{\mathrm{y}}$ components are generated as shown in Figures 1a and 1b. In a forthcoming study we will obtain synthetic spectra from accelerated particles in kinetic KHI as we did for shock simulations (Nishikawa et al. 2011, 2012).

This work is supported by NSF AST-0506666, AST-0506719, AST-0908010, and AST-0908040, NASA-NNG05GK73G, NNX07AJ88G, NNX08AG83G, NNX08 AL39G, NNX09AD16G, and NNX12AH06G. JN is supported by NCN through grant DEC-2011/01/B/ST9/03183. Simulations were performed at the Columbia facility at the NASA Advanced Supercomputing (NAS) and Kraken and Nautilus at The National Institute for Computational Sciences (NICS) which is supported by the NSF. This research was started during the program "Chirps, Mergers and Explosions: The Final Moments of Coalescing Compact Binaries" at the Kavli Institute for Theoretical Physics which is supported by the National Science Foundation under Grant No. PHY05-51164.

\section{References}

Alves, E.P., Grismayer, T., Martines, S.F., et al., 2012, ApJ, 746, L14

Giannios, D., Uzdensky, D.A., \& Begelman, M.C., 2009, MNRAS, 395, L29

Mizuno, Y., Hardee, P., \& Nishikawa, K.-I., 2007, ApJ, 662, 835

Nishikawa, K.-I., Niemiec, J., Hardee, P.E., et al., 2009, ApJ, 698, L10

Nishikawa, K.-I., Niemiec, J., Medvedev, M., et al., 2011, Adv. Space Res., 47, 1434

Nishikawa, K.-I., Niemiec, J., Zhang, B., et al., 2012, IJMP: Conf. Ser., 8, 259 
